\title{
Interactive comment on "Nocturnal new particle formation events in urban environment" by Farhad Salimi et al.
}

\section{Anonymous Referee \#1}

Received and published: 30 October 2016

The authors present an analysis of a dataset spanning more than one year of particle size distribution measurements that are analysed for new particle formation statistics and characteristics. Nighttime particle formation is an interesting phenomenon, and therefore the paper fits ACP in terms of its subject matter.

Overall, the paper is well structured, and the measurement description is good. There is a new method for determining the growth rate of particles, as well as classifying the events, which I consider very interesting. However, some missing description of the data analysis, as well as probable errors (detailed later) in the computations are problematic. I think that if these problems are corrected, the paper may well be published in ACP, but the mistakes and lack of description are so significant that they should be corrected. 
The lack of description concerns the following methods:

* line 85: The method to determine the event class is very interesting and seems promising. However, some more information is needed. How is the start time of using the regression determined? What is the meaning of the constant beta_0?

* line 100, kernel density estimation: what is meant by smoothed density of NPF events? Also, I think listing the variables (both predicted and the ones used as explanatory variables) that are handled with GAM, would be beneficial to the reader.

* The whole GAM methodology remains very unclear in the paper. Especially the validity of the model for the first thing analysed seems questionable to me: the GAM result (which I'm guessing is the line in Fig 3, top panel) seems to give different results at times 0 and 24; this makes no sense at all as the diurnal cycle should not depend of the choice of start and end times. For the annual trend the same applies. This makes the whole methodology suspect, but it is difficult to identify the problem with so little explanation given.

* $\mathrm{CS}, \mathrm{GR}$ and $\mathrm{Q}$ analysis: The obtained values seem very strange to me. The condensation sinks given (ranging from 0.5-8 $s^{\wedge}(-1)$ ) suggest that the condensing vapour lifetime is of the order of less than a second, suggesting very high concentrations of aerosol. Also, the vapour source rates are on the other hand very low, close to zero (fig 4). Unless the concentrations of aerosols are several orders of magnitude higher than usually, I think that the computations should be checked. The authors should also give in the text the variation and statistical values (mean, median, gsd, etc) of the number concentration and CS.

* Also, if the values are computed as in literature usually, it assumes a steady-state to get the formula $Q-C S \times$ C_vapour $=0$, giving $Q=C S \times$ C_vapour. C_vapour is usually taken from the growth rate $\mathrm{GR}$ by using the free molecular regime formula $\mathrm{C}$ _vapour $=A \times G R$, where $A$ is a constant. Now, if we plot these in a $x-y$ figure (as in figure 4), the axes are not independent, as the GR and CS are included in $\mathrm{Q}$ already. Therefore, 
the fits in the figures are also including dependence of these variables. This should be discussed and taken into account in the analysis.

* line 180 onwards: No clear time is given on how the daytime and nighttime were defined. This is needed for understanding the analysis.

Other comments:

Interactive

comment

* The figure captions are much too short and it is difficult to follow what is in the figure.

* figure 3: what are the lines and shaded areas?

* figure 5: what is the definition of NPF density?

* figure 9: please indicate some geographic markers (country, cities, sea, land, etc.) in the map.

One suggestion also: it would be interesting to know how the wind speed and direction affects new particle formation in the nighttime. Usually, a 'banana'-type NPF data suggests that NPF is occurring over a larger area, as advection carries air towards and away from the measurement point. However, in nighttime, wind may sometimes be non-existent. In this case, this could mean that NPF is occurring over a limited area. This could be an interesting piece of information in this case.

Interactive comment on Atmos. Chem. Phys. Discuss., doi:10.5194/acp-2016-521, 2016. 